MY APPROACH

\title{
Diagnosis and grading of dysplasia in Barrett's oesophagus
}

\section{R D Odze}

J Clin Pathol 2006;59:1029-1038. doi: 10.1136/icp.2005.035337

This review focuses on the pathological features of dysplasia in Barrett's oesophagus. Two categorisation schemes are used for grading dysplasia in the gastrointestinal tract, including Barrett's oesophagus. The inflammatory bowel disease dysplasia morphology study group system is the one most commonly used in the USA. However, some European and most far Eastern countries use the Vienna classification system, which uses the term "non-invasive neoplasia" instead of low-grade dysplasia (LGD) or high-grade dysplasia (HGD) and also uses the term "suspicious for invasive carcinoma" for lesions that show equivocal cytological or architectural features of tissue invasion. The degree of dysplasia is based on a combination of cytological and architectural atypia. However, the precise number of HGD crypts that is necessary to upgrade a biopsy from LGD to HGD has never been investigated and varies widely among expert gastrointestinal pathologists. The extent of dysplasia, particularly LGD, has also been recognised recently as an important prognostic parameter in Barrett's oesophagus. Other problematic areas of dysplasia interpretation include differentiation of regenerating epithelium versus LGD and separating HGD from carcinoma. Dysplasia associated with macroscopically visible lesions, such as ulcers, nodules or polyps, carry a high risk of synchronous or metachronous adenocarcinoma. Recently, immunostaining for $\alpha$-methylacyl-CoA-racemase has been shown to have a high degree of specificity for detection of dysplasia in Barrett's oesophagus and may be used to help distinguish negative from positive biopsies in this condition. In this review, the problematic areas in dysplasia interpretation are outlined and a specific approach to these issues is discussed.

Correspondence to: R D Odze, Brigham and Women's Hospital, Harvard Medical Śchool, 75 Francis Street, Boston, MA 02115, USA rodze@partners.org

Accepted for publication 20 November 2005
W orldwide, there are two classification systems used for dysplasia in the gastrointestinal tract (table 1) including Barrett's oesophagus. ${ }^{2}$ In 1983, the inflammatory bowel disease (IBD) dysplasia morphology study group classified dysplasia as negative, indefinite or positive (low or high grade), which is the system used most commonly in the USA. ${ }^{2}$ Recently, the World Health Organization proposed that the term "dysplasia" be replaced by "intraepithelial neoplasia", but this new term has yet to gain popularity when used in reference to preneoplastic conditions of the gastrointestinal tract. ${ }^{3}$ Some European and most far Eastern countries use the Vienna classification, but it has not gained widespread acceptance in the USA. ${ }^{1}$ The Vienna system (table 1) is similar to the one proposed by the IBD dysplasia morphology study group except that it uses the term "non-invasive neoplasia" instead of low-grade or high-grade dysplasia and also uses the term "suspicious for invasive carcinoma" for lesions that show equivocal cytological or architectural features of tissue invasion. The Vienna system was developed to reduce the widely recognised discrepancies in interpretation of dysplasia between Western and Japanese pathologists and in an effort to reach a consensus on the nomenclature of gastrointestinal neoplasia. Briefly, the basis of the differences in interpretation relates to the fact that Western pathologists define carcinoma as lesions that show histological evidence of penetration beyond the basement membrane into the lamina propria or submucosa, whereas many Japanese pathologists diagnose carcinoma on the basis of cytological changes, in conjunction with architectural changes, without the need to accurately show invasion beyond the basement membrane. Unfortunately, as outlined later, there is a high level of intraobserver and interobserver variability with regard to the pathological categorisation of dysplasia using both these systems, and particularly for epithelial lesions that show borderline features between regeneration and low-grade dysplasia (LGD). ${ }^{1} 46$ Fortunately, in both systems, the detection of clinically relevant high-grade lesions and carcinoma shows a much higher level of consistency in interpretation among pathologists.

This review focuses on my personal approach to the diagnosis and grading of dysplasia in Barrett's oesophagus, which is based on published data and on my experience as a highly referral-based and dedicated gastrointestinal pathologist with a longstanding research interest in Barrett's oesophagus. However, as a prelude to this review, it is worth noting that although morphological detection of dysplasia in mucosal biopsy specimens currently serves as the best method (biomarker) of detecting patients at increased risk of cancer in Barrett's oesophagus, it has limitations. ${ }^{71}$ One limitation relates to the inadequacy of the currently accepted definition of dysplasia as "unequivocal neoplastic epithelium confined to the basement membrane". In

Abbreviations: AMACR, $\alpha$-methylacyl-CoA-racemase; BCDA, basal crypt dysplasia-like atypia; HGD, highgrade dysplasia; IBD, inflammatory bowel disease; LGD, low-grade dysplasia; N/C ratio, nuclear/cytoplasmic ratio 
Table 1 Outline and comparison of the Vienna classification and inflammatory bowel disease (IBD) dysplasia morphology study group classification of dysplasia in IBD

\begin{tabular}{ll}
\hline Vienna & IBD study group \\
\hline $\begin{array}{l}\text { Negative for neoplasia/dysplasia } \\
\text { Indefinite for neoplasia/dysplasia }\end{array}$ & $\begin{array}{l}\text { Negative for dysplasia } \\
\text { Indefinite for dysplasia }\end{array}$ \\
$\begin{array}{l}\text { Non-invasive low-grade neoplasia (low-grade } \\
\text { adenoma/dysplasia) }\end{array}$ & $\begin{array}{l}\text { Low-grade dysplasia } \\
\text { Non-invasive high-grade neoplasia } \\
\text { High-grade adenoma/dysplasia } \\
\begin{array}{l}\text { Non-invasive carcinoma (carcinoma in situ) } \\
\text { Suspicious of invasive carcinoma }\end{array}\end{array}$ \\
$\begin{array}{ll}\text { Invasive neoplasia } \\
\text { Intramucosal adenocarcinoma } \\
\text { Submucosal carcinoma or beyond }\end{array}$ & $\begin{array}{l}\text { Adenocarcinoma* } \\
\text { Intramucosal }\end{array}$ \\
\hline *Not described by the IBD study group. & \\
\hline
\end{tabular}

fact, it is often difficult, morphologically, to recognise "atypical" epithelium as unequivocally neoplastic because of overlapping histological features with epithelial regenerative changes. Furthermore, it is also often difficult to determine, with complete certainty, whether a focus of neoplastic epithelium has, in fact, breached the basement membrane and invaded the lamina propria. Finally, there is abundant evidence to suggest that non-dysplastic (metaplastic) epithelium in Barrett's oesophagus possesses many of the biological characteristics of "neoplastic" epithelium, the nondysplastic epithelium being defined as a clonal proliferation of cells with loss of the normal capacity for regulation of cell proliferation and differentiation. ${ }^{72}$ Thus, dysplasia is a disease in need of redefinition, particularly as it relates to the development of cancer in Barrett's oesophagus.

\section{Pathological features of dysplasia in Barrett's oesophagus \\ Negative for dysplasia}

A diagnosis of "negative for dysplasia" is applied to cases that show metaplastic columnar epithelium with regenerative changes. Unfortunately, on occasion, epithelial regenerative changes may be extreme, particularly in mucosa adjacent to the neo-squamocolumnar junction or in which active inflammation or ulceration is present. In my experience, the tendency among surgical pathologists to overinterpret regenerative changes as indicative of dysplasia is often due to a lack of awareness of the wide spectrum of "atypia" that may occur in patients with Barrett's oesophagus, particularly those with persistent reflux. From a biological perspective, the progression of Barrett's oesophagus to adenocarcinoma is driven by the evolution and proliferation of clones of cells with accumulated genetic errors, a process referred to as genomic instability. ${ }^{13}{ }^{14} \mathrm{In}$ fact, the process of clonal evolution begins early in Barrett's oesophagus, before the phenotypic expression of dysplasia, when metaplastic epithelial cells acquire mutations that give them a selective proliferative advantage relative to genetically normal cells. Mutant cells containing a proliferative advantage then undergo cell division, which ultimately results in a clone of cells that contain genetic lesions identical to the original progenitor cell. In this manner, morphologically non-dysplastic Barrett's oesophagus contains progeny clones with accumulated genetic abnormalities. For instance, a recent study ${ }^{16}$ reported that $85-90 \%$ of patients with Barrett's oesophagus contain one or more pl6 mutations in non-dysplastic mucosa. In fact, mutations in the pl6 gene also correlate directly with length of columnar mucosa in Barrett's oesophagus. ${ }^{15}$ p53 mutations may also occur in patients with Barrett's oesophagus without dysplasia. ${ }^{17} 18$ These findings support the hypothesis that Barrett's oesophagus represents a neoplastic clonal proliferation rather than a polyclonal response to injury, as previously believed..$^{12}{ }^{19}$ Thus, it is not surprising that Barrett's oesophagus cases that are considered to be negative for dysplasia may show a level of atypia beyond the limits that pathologists would normally accept as part of a tissue repair reaction. In general, non-dysplastic epithelium in Barrett's oesophagus exhibits cytological features characteristic of regeneration coupled with preservation of crypt architecture (fig 1). However, a considerable degree of atypia may be present in markedly reactive epithelium. For instance, some degree of crypt budding, branching, atrophy, crowding, distortion or even cystic change may be present, especially adjacent to or underneath areas of ulceration. In addition, nuclear stratification is often present, particularly at the bases of crypts, but occasionally at the level of the surface epithelium as well, although usually to a lesser degree. Cytologically, regenerating cells contain nuclei with smooth membranes, normal nuclear/cytoplasmic $(\mathrm{N} / \mathrm{C})$ ratio, and a variable number of normal mitoses, but may also show prominent nucleoli, although without notable enlargement. In general, nuclear pleomorphism, loss of cell polarity and markedly raised N/C ratio are features of dysplasia and not of regenerating epithelium. However, newly formed epithelium covering a freshly ulcerated surface may show cells with increased N/C ratio, hyperchromaticity and slight loss of polarity and pleomorphism. In this instance, tufting of surface cells is often present, which helps declare the benign nature of the epithelium. Nevertheless, in contrast with dysplasia, goblet cells, both normal and dystrophic, are quite common. Cytoplasmic mucin may be depleted, particularly in areas of inflammation, but even in these circumstances the crypt cells usually show a progressive increase in content of mucin in epithelium close to, or at, the luminal surface. This feature, the preservation of the N/C ratio and a decrease in the degree of nuclear stratification in the upper levels of the crypts and in the surface epithelium represent the hallmark features of surface "maturation", which is the most characteristic feature of non-dysplastic epithelium in Barrett's oesophagus.

In general, nuclear and cytoplasmic changes, and stratification, are more prominent in ulcerated or actively inflamed mucosa. However, in these instances, and in contrast with dysplasia, the degree of atypia related to regenerative changes usually dissipates gradually in areas of mucosa more distant from the inflammatory infiltrate and represents a helpful feature in distinguishing it from dysplasia.

Positive for dysplasia, low and high grade

Overall, there are two general histological types of dysplasia in Barrett's oesophagus, termed "adenoma" and "nonadenoma-like" because of the resemblance, or lack thereof, of the cytological features of the dysplastic cells to sporadic colonic adenomas. However, non-conventional or unusual forms of dysplasia have been recently recognised in both Barrett's oesophagus and inflammatory bowel disease. ${ }^{20} 22$ Some of these are discussed later. "Adenomatous" LGD, which is the most common type of dysplasia in Barrett's oesophagus, shows crypts with relatively preserved architecture or perhaps only minimal distortion, and stratified atypical pencil-shaped nuclei limited, for the most part, to the basal portion of the cell cytoplasm (fig 2). The nuclei in LGD are typically elongated, crowded and hyperchromatic; they show an irregular contour and a dense chromatin pattern either with or without multiple, small inconspicuous nucleoli. Dysplastic cells are mucin depleted and show a decrease in the number of goblet cells. Other features include increased mitoses, both typical and atypical, preservation (or only slight loss) of cell polarity, increased N/C ratio, 

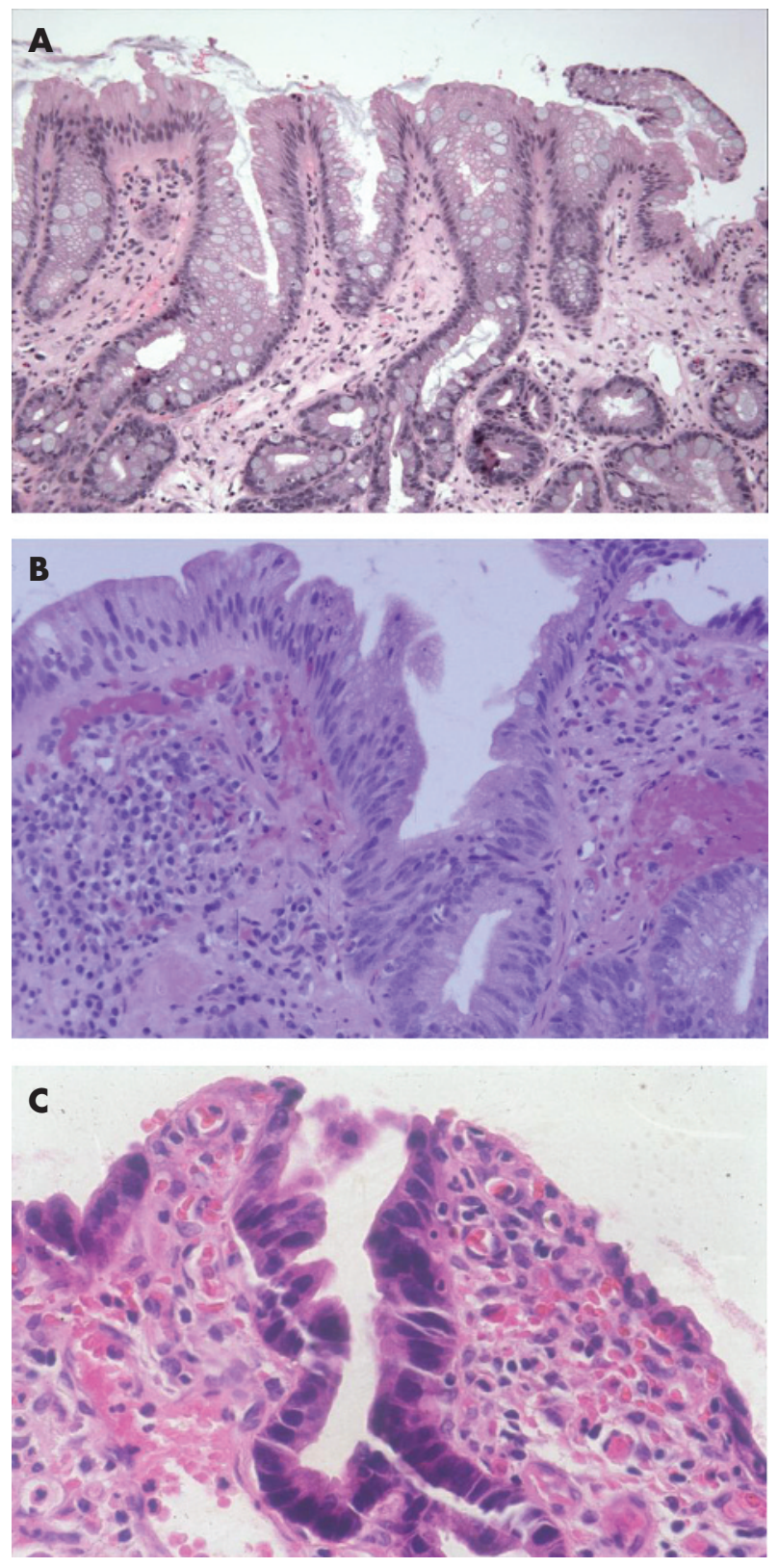

Figure 1 (A) Intestinalised epithelium in Barrett's oesophagus (BO) with mild regenerative changes. Note that a mild degree of crypt distortion, budding and crowding is a normal component of metaplastic epithelium in $\mathrm{BO}$. In one focus, there is mild stratification of the nuclei in the surface epithelium, but the nuclei are regular in shape, and the cells show a relatively low nuclear/cytoplasmic (N/C) ratio. (B) High-power photograph of marked regenerative changes in an area of mucosa close to the neosquamocolumnar junction. The epithelium shows prominent stratification of the nuclei, which includes the surface epithelium as well. A few intraepithelial neutrophils are present. Key features of regeneration in this case include tuffing of the epithelial cells, low N/C ratio, prominent nucleoli and preservation of cell polarity. (C) An area of extreme regeneration in a freshly ulcerated mucosal surface. The crypt and surface epithelium show cells with increased N/C ratio,

hyperchromaticity and mucin depletion. Note that the atypia in the monolayer of epithelium covering the freshly ulcerated surface is similar to that seen in the crypt. Biopsy specimens obtained from this area of the mucosa 6 months later showed no evidence of dysplasia.

particularly in the bases of the crypts, and lack of surface maturation, which is a characteristic feature of dysplasia in general. Most notably, dysplastic epithelium often shows an abrupt transition to non-dysplastic epithelium, which is a
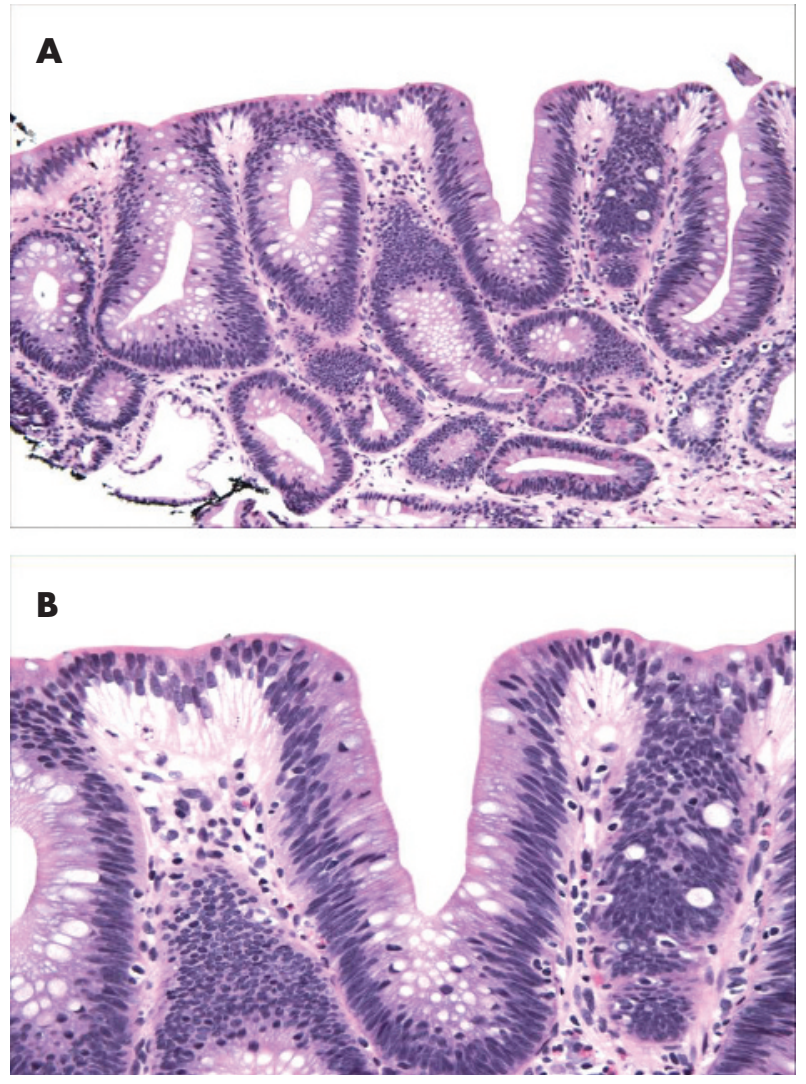

Figure 2 (A) Medium power view of low-grade dysplasia in Barrett's oesophagus. The nuclei are pencil-shaped, hyperchromatic and stratified, but limited to the basal half of the cell cytoplasm, except focally in the surface epithelium. Overall, there are minimal architectural abnormalities. At high power (B), the nuclei show clumped chromatin and inconspicuous multiple small nucleoli.

helpful feature in distinguishing dysplasia from regenerating epithelium.

With progression to high-grade dysplasia (HGD), the degree of cytological and architectural complexity becomes more advanced (table 2, fig 3). In fact, a diagnosis of HGD may be established if either of these types of abnormalities is sufficiently prominent, in the absence of the other. ${ }^{4}{ }^{18}$ Typical architectural abnormalities include crypt budding, branching, marked crowding (evident by a noticeable decrease in the amount of lamina propria between dysplastic crypts) or a villiform configuration of the surface epithelium. Crypts often have an irregular configuration and may show intraluminal papillae or bridges. However, prominent intraluminal bridges, imparting a cribriform pattern to the glands, should raise the possibility of intramucosal adenocarcinoma. Cytologically, in contrast with LGD, HGD shows cells with marked nuclear pleomorphism and irregularity of contour, increased N/C ratio, loss of cell polarity and a higher number of atypical mitoses, particularly in the upper levels of the crypts and even in the surface epithelium. Full-thickness nuclear stratification, both in the crypt and surface epithelium, is a characteristic feature of HGD. Nucleoli, when present, may be large in size and irregular in contour. Similar to LGD, mucin depletion is prominent and goblet cells, both typical and dystrophic, are markedly decreased in number or completely absent. In summary, the important features helpful in distinguishing LGD from HGD include fullthickness nuclear stratification, loss of cell polarity, particularly at higher levels of the crypts and surface epithelium, 


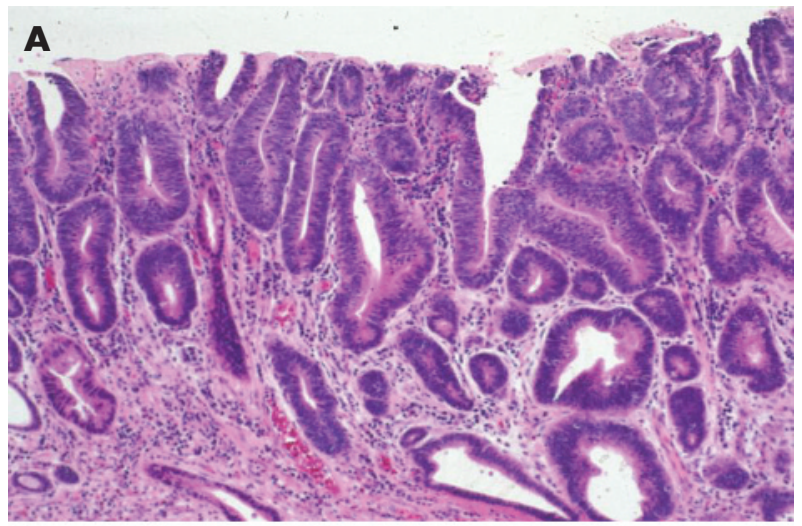

B

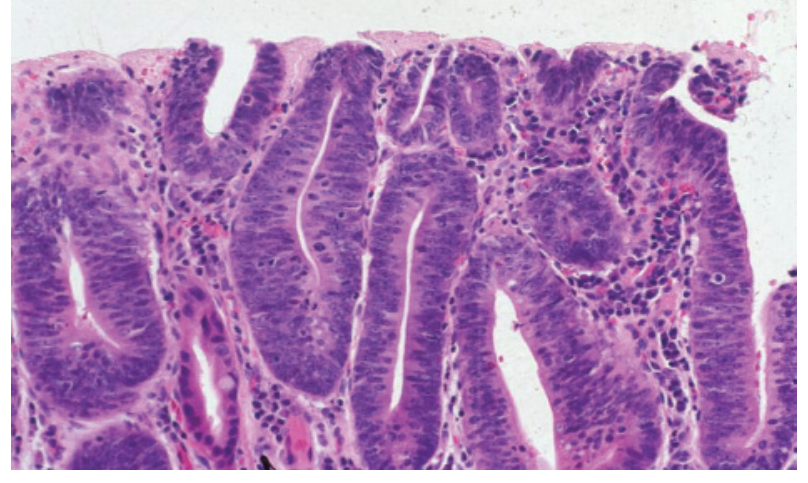

Figure 3 (A) In contrast with low-grade dysplasia (LGD), high-grade dysplasia (HGD) shows crowded crypts with more severe nuclear stratification. The nuclei reach the luminal surface in the deeper portions of the crypts. In addition, there is mild architectural distortion. On highpower view (B), the nuclei have a slightly more open chromatin pattern with prominent, sometimes multiple, nucleoli and a more marked loss of cell polarity. The nuclear/cytoplasmic ratio of HGD is markedly increased compared with LGD.

atypical mitoses or considerable architectural distortion in the HGD, as outlined earlier.

With further neoplastic progression, cells may breach the basement membrane and invade the lamina propria or muscularis mucosa, features indicative of intramucosal

Table 2 Cytological and architectural features of lowgrade and high-grade dysplasia in Barrett's oesophagus

\begin{tabular}{|c|c|c|}
\hline Feature & Low Grade & High Grade \\
\hline \multicolumn{3}{|l|}{ Cytology } \\
\hline$\uparrow \mathrm{N} / \mathrm{C}$ ratio & + & ++ \\
\hline Loss of cell polarity & - & + \\
\hline Mitosis & + & ++ \\
\hline Atypical mitosis & $+1-$ & + \\
\hline Full-thickness nuclear stratification & - & + \\
\hline Decreased goblet cells (+ / - dystrophic) & + & ++ \\
\hline Hyperchromasia & + & ++ \\
\hline Multiple nucleoli & $+1-$ & $+1-$ \\
\hline Large irregular (prominent) nucleoli & - & $+1-$ \\
\hline Irregular nuclear contour & + & ++ \\
\hline Nuclear pleomorphism & - & + \\
\hline \multicolumn{3}{|l|}{ Architecture } \\
\hline Villiform change & - & $+/-$ \\
\hline Crypt budding/branching & $+/-$ & ++ \\
\hline Crowded (back-to-back) crypts & $+1-$ & ++ \\
\hline Irregular crypt shapes & $+/-$ & + \\
\hline Intraluminal papilla/ridges & - & $+1-$ \\
\hline Lamina propria between glands & + & $+1-$ \\
\hline
\end{tabular}

adenocarcinoma (fig 4). In some institutes, patients with intramucosal adenocarcinoma are treated more aggressively (oesophageal resection) than those with HGD (aggressive surveillance and endoscopic mucosal ablation), ${ }^{723}$ and thus, distinction between these types of neoplastic lesions may be clinically important. However, in other institutes, patients with HGD or intramucosal adenocarcinoma are managed similarly. ${ }^{26} 27$ Therefore, it is important to have a clear understanding of the particular treatment regimens used at your institute before engaging in a laborious mental exercise sometimes associated with this differential diagnosis

Non-adenomatous dysplasia is rare and is a rather poorly characterised entity with regard to its biological and pathological characteristics and natural history. However, most authors consider non-adenomatous dysplasia to be a form of HGD for the purpose of patient management. Nonadenomatous dysplasia is characterised by crypts that show a prominent back-to-back gland pattern and contain cells that are more epithelioid or cuboidal-shaped, with a high N/C ratio, round or oval highly irregular-shaped nuclei, an open chromatin pattern and prominent nucleoli (fig 5). In fact, the crypts may show little or no intervening lamina propria, which often raises the possibility of intramucosal adenocarcinoma.

\section{Problematic areas of Barrett's oesophagus-associated dysplasia interpretation}

Regeneration versus low-grade dysplasia

("indefinite for dysplasia")

Although early studies suggested that patients with LGD did not show a substantially higher rate of progression to adenocarcinoma than patients with biopsies considered to be negative or indefinite for dysplasia, recent data suggest otherwise. ${ }^{7}$ Unfortunately, given the subtle gradation of changes that occur in the progression of dysplasia in Barrett's oesophagus and the wide range of morphological patterns of atypia related to regeneration and repair, there is a significant degree of intraobserver and interobserver variability in the diagnosis of dysplasia, particularly regarding cases at the lower end of the spectrum (ie, separating regeneration from dysplasia), even among experienced gastrointestinal pathologists. ${ }^{4}{ }^{6}$ The highest level of variability occurs in the differential of marked regenerative changes versus LGD, often necessitating an interim diagnosis of indefinite for dysplasia. For instance, in the original study by Reid et $a l,{ }^{4}$ regarding interobserver variation in the diagnosis

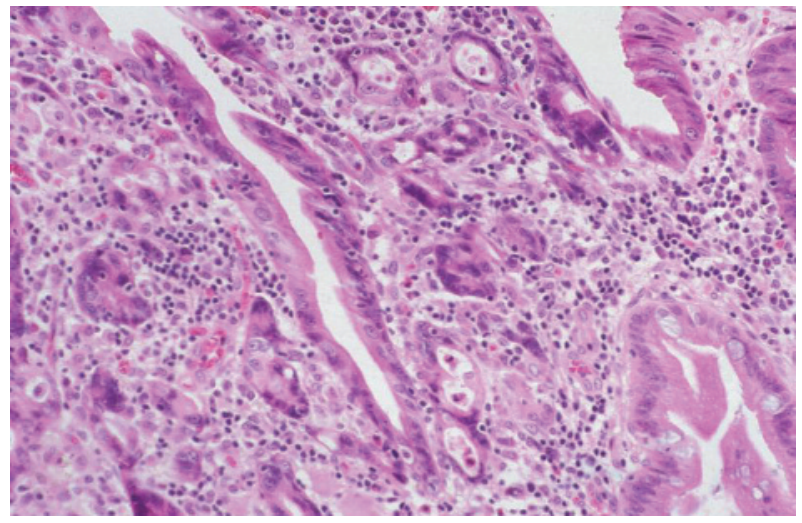

Figure 4 High-power view of high-grade dysplasia with intramucosal adenocarcinoma. The intramucosal adenocarcinoma is characterised by a proliferation of small irregular glands with markedly atypical nuclei infiltrating the lamina propria in a haphazard fashion that cannot be explained on the basis of involvement of pre-existing crypts. 

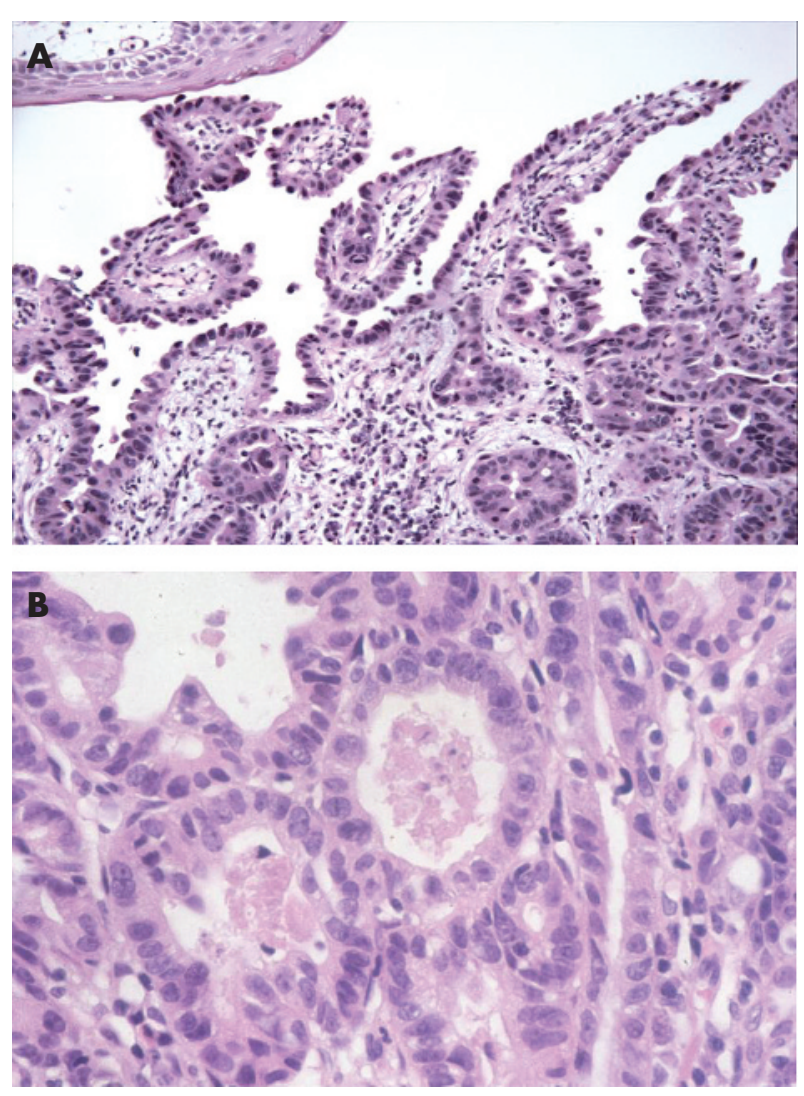

Figure 5 (A) Non-adenomatous (high-grade) dysplasia in Barrett's oesophagus. In contrast with adenomatous dysplasia, the neoplastic cells show low columnar or cuboidal phenotype, highly irregular nuclei with markedly increased nuclear/cytoplasmic (N/C) ratio and marked loss of cell polarity. High-power view of another area from the same case (B) shows a back-to-back gland pattern consisting of cuboidal cells with a high N/C ratio, prominent nucleoli and marked loss of cell polarity.

of dysplasia in Barrett's oesophagus among eight expert gastrointestinal pathologists, there was only $60 \%$ agreement in distinguishing negative from indefinite and LGD cases. In fact, in a reproducibility study by Montgomery et al, ${ }^{5}$ only slightly better agreement was noted among expert gastrointestinal pathologists in separating these categories of dysplasia. These authors also noted a high level of diagnostic difficulty in diagnosing lesions at the lower end of the dysplasia spectrum. For instance, $\kappa$ values of 0.32 and 0.15 , indicating only "fair" and "slight" agreement, were obtained for detection of LGD and indefinite for dysplasia, respectively.

In my experience, the indefinite for dysplasia category is used most often in one or more of the following three situations:

- technical issues;

- atypia related to inflammation and ulceration; and

- dysplasia-like changes present only in the bases of the crypts, with evidence of surface maturation.

Mucosal biopsy specimens that are sectioned in a tangential manner, possess marked cautery artefact or lack surface epithelium are often difficult to evaluate definitively for dysplasia (fig 6). In a study by Baak et $a^{28}$ that evaluated Barrett's oesophagus-associated biopsy specimens by morphometric analysis, 64 of 71 cases of LGD and 11 of 23 cases of HGD initially diagnosed by general pathologists were downgraded by experts on re-evaluation of the biopsy specimens. In fact, $46 \%$ of the experts' "downgrades" were thought to be due to technically inadequate tissue sections. Tangentially sectioned biopsy specimens that lack welloriented crypts and surface epithelium may be difficult to evaluate for the presence or absence of surface maturation, which is an important feature to consider in the regeneration versus dysplasia differential diagnosis.

Regenerating epithelium, particularly in the setting of active inflammation or ulceration, may, on occasion, show a considerable degree of cytological atypia similar to that in LGD or even HGD. In architecturally normal biopsy specimens with inflammation, the lack of an abrupt transition from atypical to non-atypical epithelium and the presence of surface maturation, combined with a lack of nuclear pleomorphism, atypical mitoses and loss of cell polarity, are helpful features in distinguishing regenerative changes from dysplasia. This constellation of features was also considered helpful in the interobserver study by Montgomery et al. ${ }^{5}$ In that study, the investigators thought that lesions considered to be "indefinite" showed inflammation, retention of their crypt architecture, a normal ratio of glands to lamina propria, obvious signs of surface maturation, and had nuclear changes that "approached but did not quite reach" those of LGD. In that scenario, it is reasonable to establish a diagnosis of indefinite for dysplasia, and to recommend that further biopsy specimens be obtained after aggressive reflux treatment has been instituted and the inflammation has subsided. As noted above, inflammation-induced "atypical" regenerative changes are most pronounced at the neosquamocolumnar junction, a region that shows a high level of cell turnover, injury and repair. Therefore, the diagnostic "threshold" for dysplasia should be raised when evaluating mucosal biopsy specimens from this anatomical region.

It is commonly believed among many gastrointestinal pathologists that dysplasia, regardless of the grade, is characterised by total crypt and surface epithelium involvement without surface maturation. ${ }^{245}$ This supposition is based on the commonly held belief that preneoplastic lesions, by definition, show a loss of differentiation capability. However, the author of this review has recently reported that mucosal biopsy specimens from patients with longstanding Barrett's oesophagus may, rarely, show dysplastic features that are limited to the crypt bases, without involvement of the upper portions of the crypt or surface epithelium ${ }^{20}$ (fig 7). In our study, 15 patients with Barrett's oesophagus who had "basal crypt dysplasia-like atypia (BCDA) with surface maturation" were evaluated for a variety of pathological, immunohistochemical and molecular features.

On the basis of the finding of a considerably increased association with conventional dysplasia or adenocarcinoma,

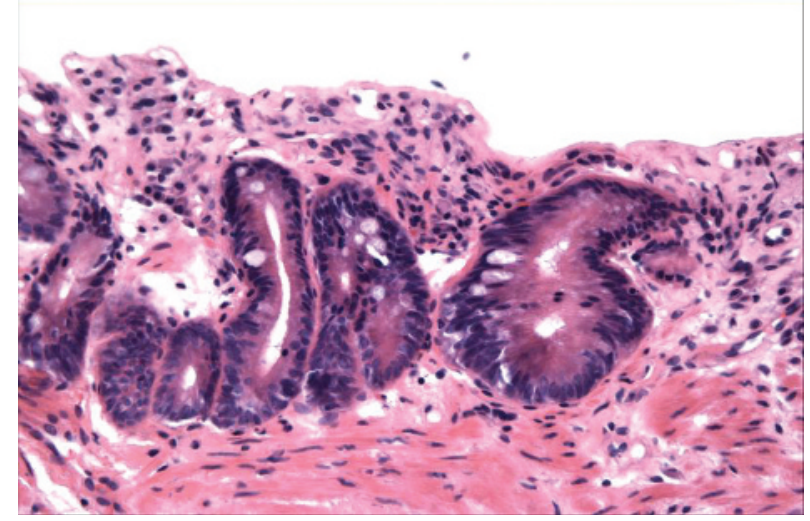

Figure 6 High-power view of an area considered indefinite for dysplasia. Although the crypts show some features of low-grade dysplasia, the absence of surface epithelium makes evaluation of surface maturation impossible. 

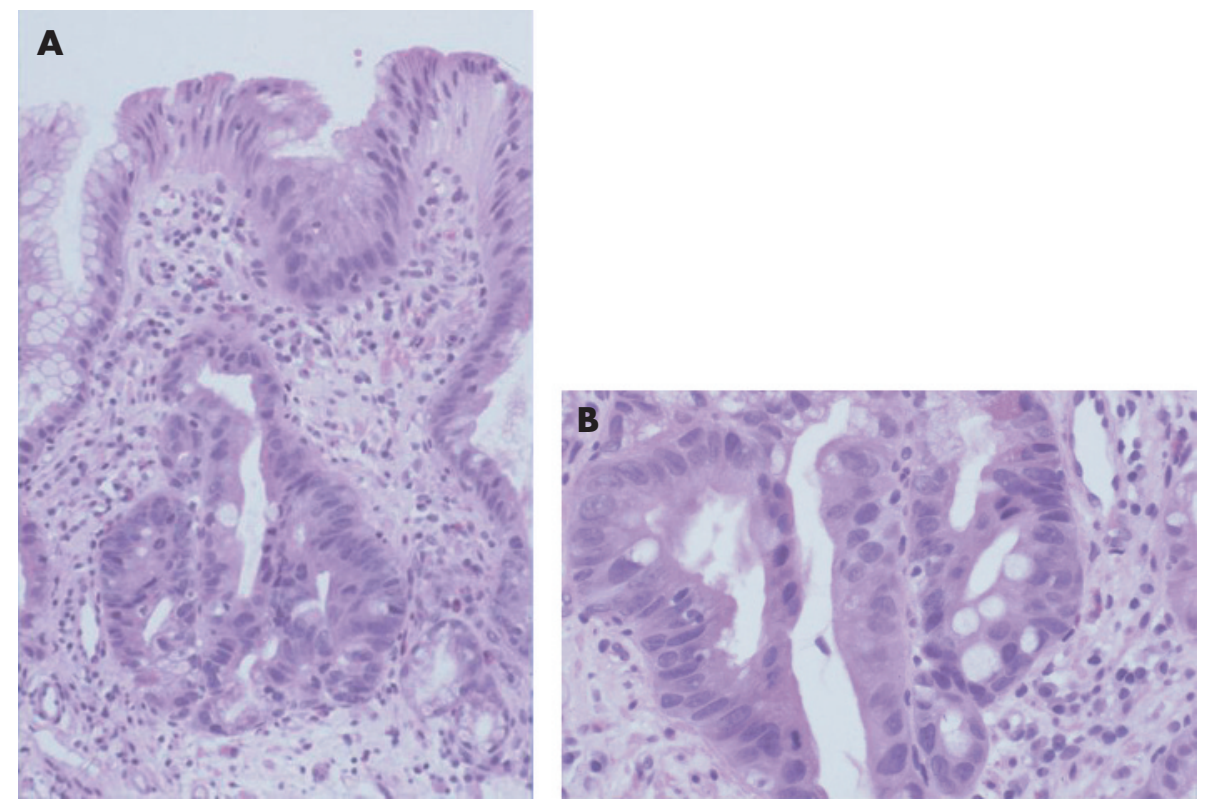

Figure 7 Medium and (A) high-power view of an area of Barrett's oesophagus (BO) showing basal crypt dysplasia-like atypia with surface maturation. In this focus, the basal portion of the crypt shows dysplastic-appearing cells characterised by nuclear enlargement, stratification, loss of polarity and a focal gland in gland pattern. Mitoses are readily identified. The superficial portion of the crypts, however, shows evidence of surface maturation characterised by a decrease in the size of nuclei, less stratification and progressive acquisition of mucin in the cytoplasm of cells on the surface $\mathrm{BO}$.

an increased prevalence rate of p53 positivity and a markedly increased rate of $9 \mathrm{pLOH}, 17 \mathrm{pLOH}$ and aneuploidy in patients with BCDA compared with controls, we concluded that this subtype of "atypical" changes probably represents true dysplasia despite the presence of surface maturation in these lesions. In fact, in the interobserver study by Montgomery et $a l^{5}$ quoted earlier, atypical lesions of this kind posed the greatest degree of diagnostic difficulty, and the widest range of diagnoses, among the participating pathologists. Of course, the presence of active inflammation in association with BCDA should prompt the pathologist to use restraint, and in this situation, it is probably wise to establish a diagnosis of indefinite for dysplasia until further biopsy specimens can be obtained after the inflammation has subsided. Figure 8 outlines a diagnostic algorithm that may be helpful when evaluating diagnostically difficult lesions in Barrett's oesophagus.

Low-grade dysplasia versus high-grade dysplasia As discussed earlier, HGD is distinguished from LGD based primarily on the basis of the degree of architectural and cytological aberrations. Unfortunately, as dysplasia progresses to cancer on a continuous scale, there are no welldefined "cut off points" that help separate these two types of lesions. In general, the overall grade of dysplasia is determined by the features of the most atypical portion of epithelium. However, the precise number of "high-grade" dysplastic crypts that are necessary to upgrade a biopsy specimen from LGD to HGD has never been investigated and varies among expert gastrointestinal pathologists. In the original classification of dysplasia in the gastrointestinal tract proposed by the IBD dysplasia morphology study group in 1983, the authors recommended, anecdotally, that "designation of a biopsy as high grade based solely on the presence of HGD in one or two crypts is probably not justified". ${ }^{2}$ In the interobserver variability study by Reid et al, ${ }^{4}$ which included many of the same authors, there was considerable disagreement regarding the minimum area required to establish a diagnosis of HGD. This issue was not discussed in the recent study on Barrett's oesophagus by Montgomery et al. ${ }^{5}$
Nevertheless, we have recent evidence to suggest that measurement of the extent of dysplasia is a highly important method of evaluating cancer risk in patients with Barrett's oesophagus. ${ }^{29}{ }^{30}$ For instance, in a recent study by Srivastava et $a l^{29}$ published in abstract form in 2005, the extent of dysplasia, regardless of the grade, was a strong risk factor for the development of invasive cancer in a 52-month follow-up study of 69 high-risk patients with Barrett's oesophagus, 28 of whom eventually developed adenocarcinoma. In that study, the mean number of crypts with LGD in patients who ultimately developed adenocarcinoma was 5.8 compared with only 2.6 in patients without cancer. These results are supported by a study by Buttar et $a l^{30}$ in which the extent of HGD in Barrett's oesophagus was shown to be correlated with the risk of developing adenocarcinoma. In their study, cancer-free survival rates were considerably higher in patients with "focal HGD" than in those with "diffuse HGD". However, a study by Dar et al, ${ }^{31}$ in which the extent of HGD was evaluated on the basis of the number of levels of the oesophagus that contained mucosa with HGD, did not corroborate the results of Buttar et al. ${ }^{30}$ In my practice, I always try to provide an objective measurement of the extent of LGD and HGD in all biopsy specimens with dysplasia. Although anecdotal, clinicians find this information useful when planning treatment strategies for patients with Barrett's oesophagus. For instance, a biopsy sign out indicating that there is "LGD and focal HGD" (implying that HGD includes only a small proportion of the number of dysplastic crypts) provides additional objective information and may prompt more aggressive surveillance or alternative protocol treatments rather than oesophagectomy, which is still the most commonly applied method of treatment for patients having Barrett's oesophagus with HGD.

\section{High-grade dysplasia versus intramucosal adenocarcinoma}

As mentioned earlier, the distinction between HGD and intramucosal adenocarcinoma may be difficult and is often clinically relevant (fig 9). Lymphatic vessels are present in the 


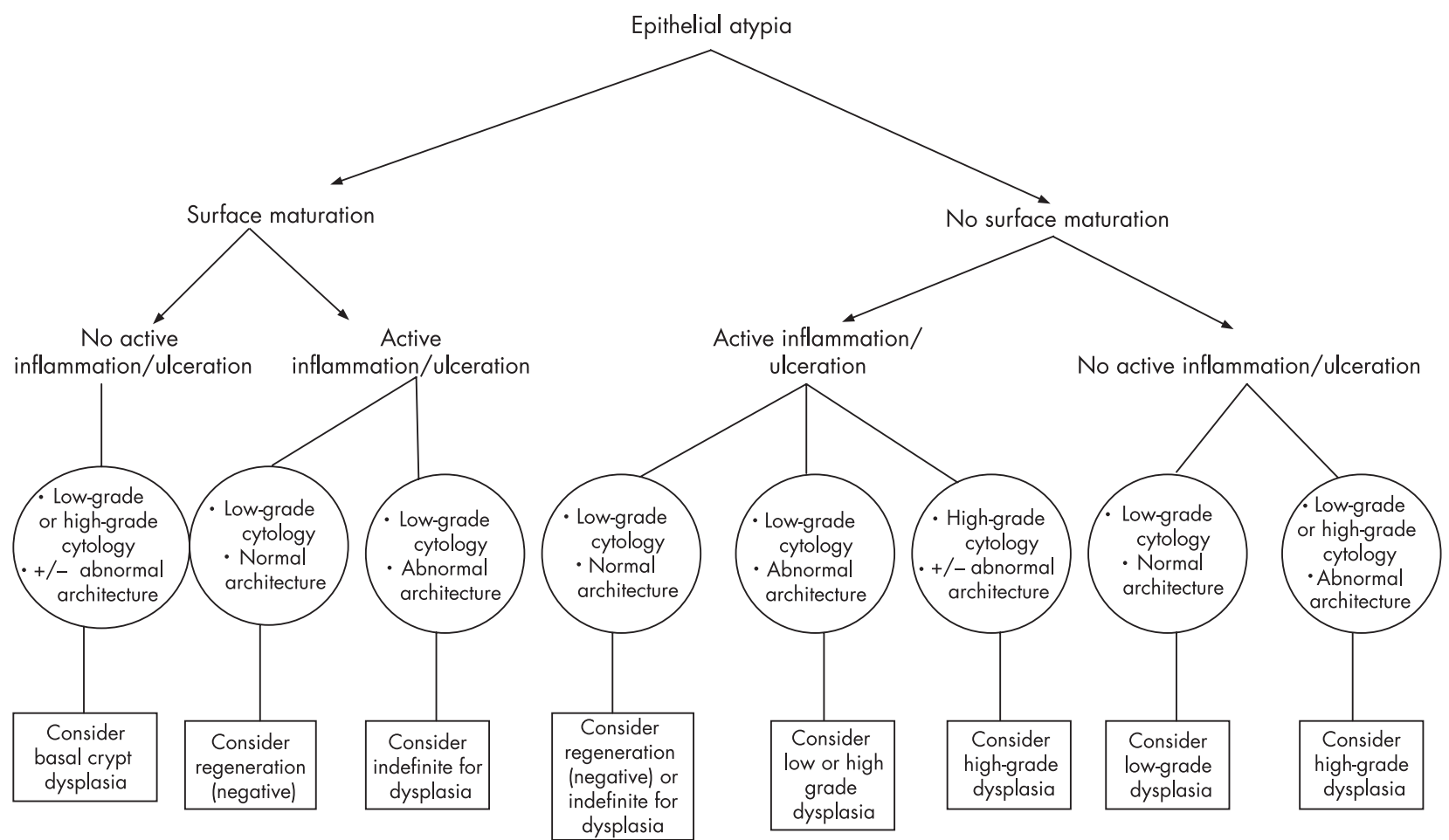

Figure 8 Diagnostic algorithm for the differential diagnosis of epithelial atypia in Barrett's oesophagus.

lamina propria of the oesophageal mucosa. As a result, there is a $5-8 \%$ risk of lymph node metastases in patients with tumours limited to the mucosal compartment, which is a major reason why oesophagectomy is widely regarded as the best method of treatment for patients having Barrett's oesophagus with intramucosal adenocarcinoma. ${ }^{23} 2632$ In contrast, some studies suggest that a vigorous surveillance biopsy protocol is a practical and safe alternative to oesophagectomy for patients with HGD. ${ }^{24}{ }^{25}$ In a study by Ormsby et al, ${ }^{23} 75$ oesophagectomy specimens were reviewed by two gastrointestinal pathologists and one general surgical pathologist and classified as either, intramucosal or submucosal adenocarcinoma. ${ }^{23}$ The level of interobserver agreement for HGD versus intramucosal adenocarcinoma was only fair $(\kappa=0.42)$ and, most importantly, did not improve greatly after establishment of uniform histological criteria $(\kappa=0.5)$. In my experience, there is a tendency for general pathologists to overdiagnose intramucosal adenocarcinoma. Often, this is because prominent architectural aberrations, such as crypt budding and branching, are interpreted as evidence of invasion, rather than simply complex in situ disease. My personal practice is to diagnose intramucosal carcinoma only when one or more of the following architectural features are present:

- single cells or small clusters of tightly compact back-toback glands are present in the lamina propria

- there is a complex gland-in-gland or "cribriforming" pattern present, with evidence of an expansion of the lamina propria and distortion of the surrounding crypts

- neoplastic cells or glands are present that show a back-toback or highly irregular architectural glandular arrangement, which cannot be explained by the presence of pre-existing Barrett's glands, as previously defined by Ormsby et al. ${ }^{23}$

The presence of necrosis or desmoplasia is evidence in favour of adenocarcinoma as well, although these features are rarely present in carcinomas limited to the mucosa, and, may not be present in submucosal invasive carcinomas either. ${ }^{23}$ When in doubt, I advocate using the term "HGD with foci suspicious, but not diagnostic, of intramucosal adenocarcinoma" (which corresponds to category 4.3 in the Vienna Classification System) and recommend that repeat biopsies should be performed if this distinction is clinically relevant.

Intramucosal versus submucosal adenocarcinoma On occasion, dysplastic glands may be located in the muscularis mucosa (fig 10). In this situation, the differentiation of "misplaced" non-invasive glands from truly invasive glands may be difficult. We must be cautious not to overinterpret these findings, particularly if the glands contain cytologically bland or only LGD changes, and the crypts are

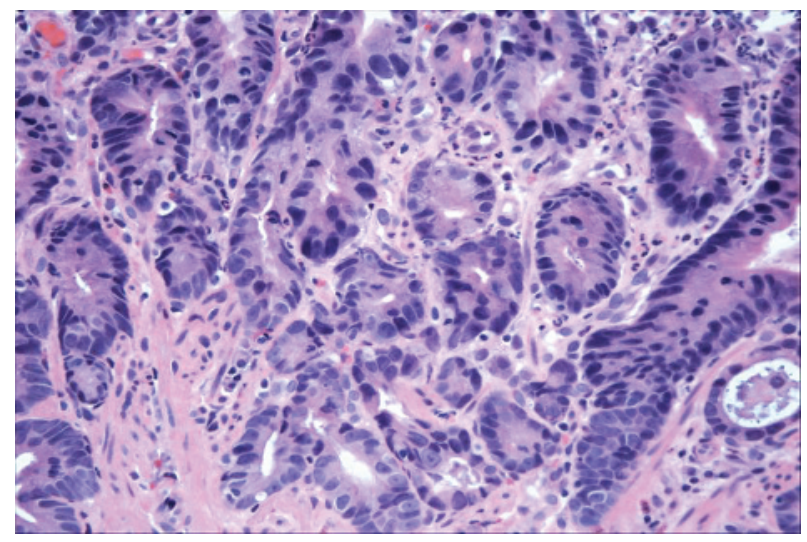

Figure 9 High-grade dysplasia in Barrett's oesophagus with a focal area highly suggestive of intramucosal adenocarcinoma. In the centre of the field is a proliferation of small glands in a back-to-back configuration, with little intervening lamina propria, which is difficult to explain on the basis of involvement of pre-existing crypts. There is a suggestion of isolated single cells in the lamina propria as well. 
not particularly distorted in shape. One reason for exercising caution is that patients with Barrett's oesophagus often develop a new (superficial), more luminally situated, layer of muscularis mucosa that directly underlies the region of metaplastic columnar epithelium. ${ }^{33}$ Unfortunately, the nature of the stroma that lies between the original (deep) and the new muscularis mucosa has never been investigated. As a result, it also remains unclear whether glands that penetrate through the new muscularis mucosa should be considered to be intramucosal or submucosal carcinomas. However, in a recent study of 120 patients with early adenocarcinoma (13 with HGD and 107 with stage Tl adenocarcinoma) of the oesophagus or gastroesophageal junction treated by oesophagectomy in which outcome was stratified according to the depth of tumour invasion, patients with tumours invading the "new" muscularis mucosa had a similar outcome as those with invasion into the original (deep) muscularis. ${ }^{34}$ Only $1 \%$ of these patients had lymph node metastasis compared with $44 \%$ of the patients with tumours that penetrated into the mid or deep portion of the original ("true") submucosa. These data suggest that the stromal space located between the original and new muscularis mucosa represents "lamina propria" and tumours that infiltrate this region should probably be considered intramucosal, from a biological point of view. Nevertheless, further studies are needed in this regard.

\section{Dysplasia and macroscopically visible lesions}

Several studies have shown that the natural history and risk of malignancy in Barrett's oesophagus are highly dependent on the macroscopic features of the dysplastic lesions. ${ }^{35} 39$ As a result, the diagnosis and grading of dysplasia in Barrett's oesophagus should be carried out in conjunction with knowledge of the endoscopic features of the patient. Common macroscopic lesions include ulcers, nodules (defined as an area of subtle mucosal elevation measuring $<\mathrm{lcm}$ in diameter) and strictures. In a study by Hillman et al, ${ }^{36}$ patients with one or more macroscopic lesions were more likely to develop HGD and cancer than those without endoscopically identifiable lesions. In a recent study by Buttar et al, , $^{30} 15$ of 25 (60\%) patients with dysplastic nodules had cancer compared with only 17 of $25(23 \%)$ patients without nodules. In fact, the risk of cancer was increased by a factor of 4 in patients who had visible nodules at endoscopy. In a study by Montgomery et $a l^{35}$ a higher proportion of patients with Barrett's oesophagus with ulcers had HGD or adenocarcinoma than patients without ulcers, and the

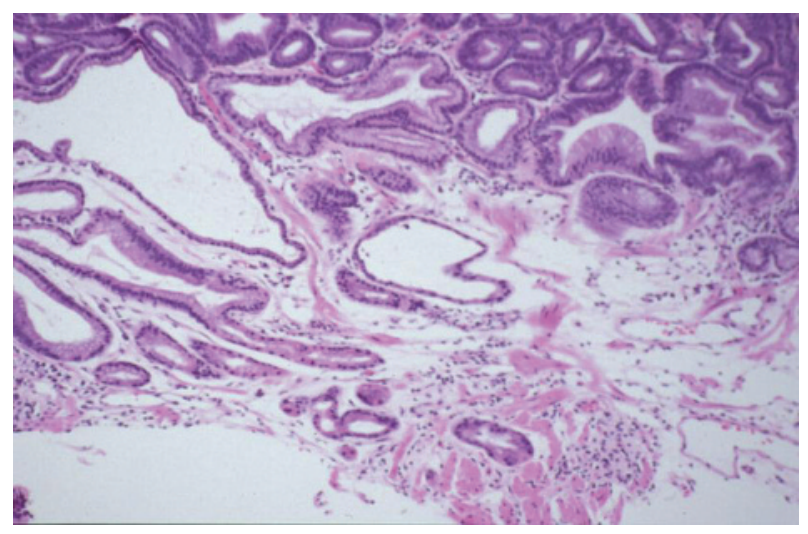

Figure 10 Mucosal biopsy from a patient with Barrett's oesophagus (BO) showing low-grade dysplasia and the presence of atypical glands in the superficial muscularis mucosa. The glands in the deeper portions of the mucosa show an attenuated epithelium. However, this is not uncommon in $\mathrm{BO}$ and should not be interpreted as evidence of adenocarcinoma. presence of an ulcer with HGD increased the likelihood of detecting carcinoma in a subsequent resection specimen. Mucosal nodularity offers the opportunity for endoscopic mucosal resection and the capability of assessing more tissue than endoscopic biopsy samples.

Rarely, dysplasia in Barrett's oesophagus may grow as a well-defined polyp with an adenoma-like appearance. ${ }^{37} 38$ However, owing to the previously reported strong association of these lesions with HGD and adenocarcinoma, and with dysplasia in the surrounding flat dysplasia, these lesions should be considered "polypoid dysplasia" rather than "adenoma". ${ }^{37}$ The term adenoma imparts a "benign" connotation to the lesion and may lead to undertreatment by polypectomy rather than oesophagectomy.

\section{Adjunctive markers in the diagnosis of dysplasia}

Many studies have evaluated the potential utility of immunohistochemical or molecular markers as adjunctive methods in detecting dysplasia and in distinguishing reactive from dysplastic epithelium in Barrett's oesophagus. ${ }^{71} 1628$ 40-49 Most of the markers investigated have been linked, in some capacity, to the pathogenesis of cancer and include those associated with control of cell proliferation, intercellular adhesion and tumour suppression, among others. Of the vast array of markers evaluated, detection of DNA content abnormalities by flow cytometry (aneuploidy or elevated $4 \mathrm{~N}$ fraction) and evaluation of mutations or loss of homozygosity of the pl6 and $p 53$ genes are the most promising potential methods to help identify high-risk patients. ${ }^{750}$ Unfortunately, none of these methods has been shown to be particularly useful in differentiating non-dysplastic from dysplastic epithelium in routine pathology practice. Other methods, such as computerised quantitative pathology or measurement of proliferation indices, have also been shown to decrease diagnostic variability in this regard, but these methods have not been standardised. ${ }^{28}{ }^{41}$ Evaluation of $p 53$ by immunostaining remains the most controversial. ${ }^{73} 51$

Proponents of $p 53$ immunostaining as an adjunctive diagnostic method state that the $p 53$ gene is only rarely mutated in non-dysplastic epithelium and that the frequency of mutations has been shown to increase dramatically in HGD and adenocarcinoma. Unfortunately, $p 53$ overexpression by immunohistochemistry can be detected in up to $10 \%$ of cases considered to be morphologically negative for dysplasia. ${ }^{43} 5153$ In addition, several studies have shown a high rate of false positive staining (up to 56\%) in the absence of $p 53$ mutations, and a high frequency of false negative staining as well (up to $30 \%$ ). ${ }^{43}$ Non-specific binding of $p 53$ to non- $p 53$ mutation-related antigens may also lead to false positive results. Furthermore, $p 53$ results may vary substantially depending on the specific type of antibody used. In fact, certain types of mutations result in the production of a p53 protein that does not bind to some antibodies directed against the wild-type protein. ${ }^{43}$ Finally, no known antibody, or combination of antibodies, can detect all $p 53$ mutations. For these reasons, I do not advocate the use of $p 53$ immunostaining in routine practice to help differentiate regenerating from dysplastic epithelium in diagnostically difficult cases.

However, recently, immunostaining for $\alpha$-methylacyl-CoAracemase (AMACR), an antibody often used in the assessment of diagnostically difficult atypical and potentially neoplastic lesions of the prostate, has been shown to have a high degree of specificity for detection of dysplasia in Barrett's oesophagus and inflammatory bowel disease. ${ }^{45}$ In a recent study by Dorer et al, ${ }^{45}$ AMACR was not detected in any of 36 cases with Barrett's oesophagus without dysplasia ( $0 \%$ ) but was positive in $38 \%, 81 \%$ and $72 \%$ of cases of LGD, HGD and adenocarcinoma, respectively. In that study, three 
"indefinite" cases were also positive, but in the one case in which follow-up information was available, adenocarcinoma was detected on follow-up. Thus, AMACR immunostaining may represent a potentially useful adjunctive method in differentiating reactive from dysplastic epithelium in Barrett's oesophagus, but, in my opinion, requires validation by other investigators before use in routine clinical practice.

\section{SUMMARY}

Despite the problems associated with detection and reproducibility, morphological evaluation of dysplasia in mucosal biopsy specimens still remains the mainstay of surveillance and treatment of Barrett's oesophagus worldwide. Careful attention to the cytological and architectural features of dysplasia, as outlined earlier, and recognition of the extreme degrees of regeneration that may occur in this condition, can help minimise observer error. Use of consultants with particular expertise in gastrointestinal pathology is highly recommended before institution of definitive management in patients with Barrett's oesophagus with "atypical" biopsy specimens. Fortunately, pathologists have a reasonably good track record at detecting clinically relevant dysplasia, such as HGD and intramucosal adenocarcinoma, lesions that usually necessitate aggressive methods of intervention such as endoscopic mucosal resection, mucosal ablation or surgical resection. Adjunctive diagnostic methods, such as AMACR immunostaining, are promising, but further studies are needed to find either non-morphology-based or morphology-based reproducible methods that can reliably help stratify cancer risk in patients with Barrett's oesophagus.

Competing interests: None declared.

\section{REFERENCES}

1 Schlemper RJ, Riddell RH, Kato Y, et al. The Vienna classification of gastrointestinal epithelial neoplasia. Gut 2000;47:251-5.

2 Riddell RH, Goldman H, Ransohoff DE, et al. Dysplasia in inflammatory bowel disease: standardized classification with provisional clinical information. Hum Pathol 1983; 14:931-68

3 Werner M, Flejou JF, Hanaut P, et al. Adenocarcinoma of the oesophagus. In: Hamilton R, Aaltonen LA, eds. Pathology and genetics of tumours of the digestive system. Lyon: IARC Press, 2000:20-26.

4 Reid BJ, Haggitt RC, Rubin EC, et al. Observer variation in the diagnosis of dysplasia in Barrett's esophagus. Hum Pathol 1988;19:166-78.

5 Montgomery E, Bronner MP, Goldblum JR, et al. Reproducibility of the diagnosis of dysplasia in Barrett esophagus: a reaffirmation. Hum Pathol $2001 ; 32: 368-78$

6 Montgomery E. Is there a way for pathologists to decrease interobserver variability in the diagnosis of dysplasia? Arch Pathol Lab Med 2005; 129:174-6.

7 Reid BJ, Blount PL, Rabinovitch PS. Biomarkers in Barrett's esophagus. Gastrointest Endoscopy Clin N Am 2003;13:369-97.

8 Macdonold CE, Wicks AC, Playford RJ. Final results from 10-year cohort of patients undergoing surveillance for Barrett's esophagus: observational study. BMJ 2000;321:1252-155

9 Montgomery E, Goldblum JR, Greenson JK, et al. Dysplasia as a predictive marker for invasive carcinoma in Barrett's esophagus: a follow-up study based on 138 cases from a diagnostic variability study. Hum Pathol 2001;32:379-88

10 Skacel M, Petras RE, Gramlich TL, et al. The diagnosis of low-grade dysplasia in Barrett's esophagus and its implications for disease progression. Am J Gastroenterol 2000:95:3383-7.

11 Sharma P, McQuaid K, Dent J, et al. A critical review of the diagnosis and management of Barrett's esophagus: the AGA Chicago Workshop. Gastroenterology 2004;127:310-30.

12 Maley CC, Galipeau PC, Li X, et al. Selectively advantageous mutations and hitchhikers in neoplasms: pl6 lesions are selected in Barrett's esophagus. Cancer Res 2004;64:3414-27.

13 Barrett MT, Sanchez CA, Prevo L, et al. Evolution of neoplastic cell lineages in Barrett oesophagus. Nat Genet 1999;22:106-9.

14 Nowell PC. The clonal evolution of tumor cell populations. Science 1976; 194:23-8.

15 Wong DJ, Paulson TG, Prevlo U, et al. pl6 INK $4_{a}$ lesions are common, early abnormalities that undergo clonal expansion in Barrett's metaplastic epithelium. Cancer Res 2001;61:8284-9.

16 Bian YS, Ostorheld MC, Fontolliet C, et al. pl6 inactivation by methylation of the CDKN2A promoter occurs early during neoplastic progression in Barrett's esophagus. Gastroenterology 2002;122:1113-1121.
17 Campomenosi $\mathbf{P}$, Conio $M$, Bogliolo $M$, et al. p53 is frequently mutated in Barrett's metaplasia of the intestinal type. Cancer Epidemiol Biomarkers Prev 1996;5:559-65.

18 Reid BJ, Prevo U, Galipeau PC, et al. Predictors of progression in Barrett's esophagus II: baseline 17p (p53) loss of heterozygosity identifies a patient subset at increased risk for neoplastic progression. Am J Gastroenterol $2001 ; 96: 2839-48$

19 Reid BJ, Barrett MT, Galipeau PC, et al. Barrett's esophagus: ordering the events that lead to cancer. Eur J Cancer Prev 1996;5(Suppl):57-65.

20 Lomo LC, Bloun PL, Sanchez CA, et al. Crypt dysplasia with surface maturation: a clinical, pathologic and molecular study of a Barrett's esophagus cohort. Am J Surg Pathol 2006;30:423-35.

21 Rubio CA, Befrits R, Jaramillo E, et al. Villous and Serrated Adenomatous Growth Bordering Carcinomas in Inflammatory Bowel Disease. Anticancer Res 2000;20:4761-4.

22 Anderson SN, Lovig T, Clausen OPF, et al. Villous, hypermucinous mucosa in long standing ulcerative colitis shows high frequency of K-ras mutations. Gut 1999:45:686-92.

23 Ormsby $\mathbf{A H}$, Petras RE, Henricks WH, et al. Observer variation in the diagnosis of superficial oesophageal adenocarcinoma. Gut 2002;51:671-6.

24 Levine DS, Haggitt RC, Blount PL, et al. An endoscopic biopsy protocol can differentiate high-grade dysplasia from early adenocarcinoma in Barrett's esophagus. Gastroenterology 1993;105:40-50.

25 Reid BJ, Weinstein WM, Lewin KJ, et al. Endoscopic biopsy can detect highgrade dysplasia or early adenocarcinoma in Barrett's esophagus without grossly recognizable neoplastic lesions. Gastroenterology 1988;94:81-90.

26 Rice TW, Falk GW, Achkar E, et al. Surgical management of high-grade dysplasia in Barrett's esophagus. Am J Gastroenterol 1993;88:1832-6.

27 Pera M, Trastek VF, Carpenter HA, et al. Barrett's esophagus with high-grade dysplasia: an indication for esophagectomy? Ann Thorac Surg 1992;54:199-203.

28 Baak JPA, ten Kate FJW, Offerhaus GJA, et al. Routine morphometrical analysis can improve reproducibility of dysplasia grade in Barrett's oesophagus surveillance biopsies. J Clin Pathol 2002;55:910-6.

29 Srivastava A, Hornick JL, Blount PL, et al. Extent of low-grade dysplasia is a risk factor for cancer in Barrett's esophagus. Gastroenterology 2005; 128:A240.

30 Buttar NS, Want KK, Sebo TJ, et al. Extent of high-grade dysplasia in Barrett's esophagus correlates with risk of adenocarcinoma. Gastroenterology 2001;120:1630-9

31 Dar MS, Goldblum JR, Rice TW, et al. Can extent of high-grade dysplasia in Barrett's oesophagus predict the presence of adenocarcinoma at oesophagectomy? Gut 2003;52:486-9.

32 Bogomoletz WV, Molas G, Gatey B, et al. Superficial squamous cell carcinoma of the esophagus: a report of 76 cases and review of the literature. Am J Surg Pathol 1989; 13:535-46.

33 Takubo K, Sasajima K, Yamashita K, et al. Double muscularis mucosae in Barrett's esophagus. Hum Pathol 1991;22:1158-61.

34 Westerterp M, Koppert LB, Buskens CJ, et al. Outcome of surgical treatment for early adenocarcinoma of the esophagus or gastro-esophageal junction. Virchows Arch 2005;446:497-504.

35 Montgomery E, Bronner MP, Greenson MD, et al. Are ulcers a marker for invasive carcinoma in Barrett's esophagus? Data from a diagnostic variability study with clinical follow-up. Am J Gastroenterol 2002;97:27-31.

36 Hillman LC, Chiragakis L, Clarke AC, et al. Acid peptic disease and complication: Barrett's esophagus: macroscopic markers and the prediction of dysplasia and adenocarcinoma. J Gastroenterol Hepatol 2003; 18:526-33.

37 Thurberg BL, Duray PH, Odze RD. Polypoid dysplasia in Barrett's esophagus: a clinicopathologic, immunohistochemical, and molecular study of five cases. Hum Pathol 1999;30:745-52.

38 Arnold GL, Mardini HE. Barrett's esophagus-associated polypoid dysplasia: a case report and review of the literature. Dig Dis Sci 2002;47:1897-900.

39 Reid BJ, Blount PL, Feng Z, et al. Optimizing endoscopic biopsy detection of early cancers in Barrett's high-grade dysplasia. Am J Gastroenterol 2000:95:3089-96.

40 Skacel M, Petras RE, Rybicki LA, et al. p53 expression in low-grade dysplasia in Barrett's esophagus: correlation with interobserver agreement and disease progression. Am J Gastroenterol 2002;97:2508-13.

41 van Sandick JW, Baak JPA, van Lanschot JJB, et al. Computerized quantitative pathology for the grading of dysplasia in surveillance biopsies of Barrett's oesophagus. J Pathol 2000;190:177-83.

42 Lorinc E, Jakobsson B, Landberg G, et al. Ki67 and p53 immunohistochemistry reduces interobserver variation in assessment of Barrett's oesophagus. Histopathology 2005;46:642-8

43 Ireland AP, Clark GWB, DeMeester TR. Barrett's esophagus: the significance of p53 in clinical practice. Annals Surg 1997;225:17-30.

44 Younes M, Lebovitz RM, Lechago LV, et al. p53 protein accumulation in Barrett's metaplasia, dysplasia, and carcinoma: a follow-up study. Gastroenterology 1993; 105:1637-42.

45 Dorer RK, Glickman JN, Blount PL, et al. Immunostaining for AMACR is useful in distinguishing reactive from dysplastic epithelium in Barrett's esophagus and inflammatory bowel disease. Am J Surg Pathol 2006;7:871-7.

46 Hong MK, Laskin WB, Herman BE, et al. Expansion of the Ki-67 proliferative compartment correlates with degree of dysplasia in Barrett's esophagus. Cancer 1995;75:423-9.

47 Hansel DE, Dhara S, Huang RC, et al. CDC2/CDK1 expression in esophageal adenocarcinoma and precursor lesions serves as a diagnostic and cancer progression marker and potential novel drug target. Am J Surg Pathol 2005;29:390-9.

48 Bailey T, Biddlestone L, Shepherd N, et al. Altered cadherin and catenin complexes in the Barrett's esophagus-dysplasia-adenocarcinoma sequence: 
correlation with disease progression and dedifferentiation. Am J Pathol $1998 ; 152: 135-44$.

49 Weston AP, Banerjee SK, Sharma P, et al. p53 protein overexpression in low grade dysplasia (LGD) in Barrett's esophagus: immunohistochemical marker predictive of progression. Am J Gastroenterol 2001;96:1355-62.

50 Reid BJ, Levine DS, Longton G, et al. Predictors of progression to cancer in Barrett's esophagus: baseline histology and flow cytometry identify low- and high-risk patients subsets. Am J Gastroenterol 2000;95:1669-76.
51 Greenblatt MS, Bennett WP, Hollstein MC, et al. Mutations in the p53 tumor suppressor gene: clues to cancer etiology and molecular pathogenesis. Cancer Res 1994;54:4855-78.

52 Jones DR, Davidson AG, Summers CL, et al. Potential application of p53 as an intermediate biomarker in Barrett's esophagus. Ann Thorac Surg 1994;57:598-603.

53 Ramel S, Reid BJ, Sanchez CA, et al. Evaluation of $\mathrm{p} 53$ protein expression in Barrett's esophagus by two-parameter flow cytometry. Gastroenterology 1992;102:1220-8.

\section{Clinical Evidence-Call for contributors}

Clinical Evidence is a regularly updated evidence-based journal available worldwide both as a paper version and on the internet. Clinical Evidence needs to recruit a number of new contributors. Contributors are healthcare professionals or epidemiologists with experience in evidence-based medicine and the ability to write in a concise and structured way.

Areas for which we are currently seeking contributors:

- Pregnancy and childbirth

- Endocrine disorders

- Palliative care

- Tropical diseases

We are also looking for contributors for existing topics. For full details on what these topics are please visit www.clinicalevidence.com/ceweb/contribute/index.jsp

However, we are always looking for others, so do not let this list discourage you.

Being a contributor involves:

- Selecting from a validated, screened search (performed by in-house Information Specialists) epidemiologically sound studies for inclusion.

- Documenting your decisions about which studies to include on an inclusion and exclusion form, which we keep on file.

- Writing the text to a highly structured template (about 1500-3000 words), using evidence from the final studies chosen, within 8-10 weeks of receiving the literature search.

- Working with Clinical Evidence editors to ensure that the final text meets epidemiological and style standards.

- Updating the text every 12 months using any new, sound evidence that becomes available. The Clinical Evidence in-house team will conduct the searches for contributors; your task is simply to filter out high quality studies and incorporate them in the existing text.

If you would like to become a contributor for Clinical Evidence or require more information about what this involves please send your contact details and a copy of your CV, clearly stating the clinical area you are interested in, to CECommissioning@bmigroup.com.

\section{Call for peer reviewers}

Clinical Evidence also needs to recruit a number of new peer reviewers specifically with an interest in the clinical areas stated above, and also others related to general practice. Peer reviewers are healthcare professionals or epidemiologists with experience in evidence-based medicine. As a peer reviewer you would be asked for your views on the clinical relevance, validity, and accessibility of specific topics within the journal, and their usefulness to the intended audience (international generalists and healthcare professionals, possibly with limited statistical knowledge). Topics are usually 1500-3000 words in length and we would ask you to review between 2-5 topics per year. The peer review process takes place throughout the year, and out turnaround time for each review is ideally 10-14 days. If you are interested in becoming a peer reviewer for Clinical Evidence, please complete the peer review questionnaire at www.clinicalevidence.com/ceweb/contribute/peerreviewer.jsp 